\title{
CHECK-LIST OF ANDALUSIA (S. SPAIN) SEAWEEDS. III. RHODOPHYCEAE
}

\author{
Francisco CONDE, Antonio FLORES-MOYA, Juan SOTO, \\ María ALTAMIRANO \& Alberto SÁNCHEZ
}

\begin{abstract}
Check-list of Andalusia (S. Spain) seaweeds. III. Rhodophyceae. A check-list of the Rhodophyceae from the Andalusian coast, based on literature records (from 1807 to 1996), is presented. Taxa are listed by provinces. The catalogue contains 348 species and infraspecific taxa under current taxonomy.
\end{abstract}

Key words. Andalusia, Rhodophyceae, Check-list, Iberian Peninsula, seaweeds.

RESUMEN. Catálogo de las Rhodophyceae de Andalucía (S. España). Se presenta el listado de las Rhodophyceae de las costas andaluzas a partir de datos bibliográficos (desde 1807 hasta 1996). Los taxa se desglosan por provincias. El catálogo contiene 348 especies y taxa infraespecíficos.

Palabras clave. Andalucía, Rhodophyceae, listado, macroalgas marinas, Península Ibérica.

\section{INTRODUCTION}

This paper presents a check-list of Rhodophyceae from Andalusian coasts, similar in scope to those previously published dedicated to Phaeophyceae (Flores-Moya et al., 1995a) and Chlorophyceae (Flores-Moya et al., 1995b). The present paper brings the base data of taxa ur to date, standardizing the taxonomic treatment used by previous authors.

\section{MATERIALS AND METHODS}

The presence of each taxon is indicated by political province of Andalusia: Huelva (HU), Cádiz (CA), Málaga (MA), Granada (GR), and Almería (AL) (see map in Flores Moya et al., 1995a). Italics have been used for accepted taxa and Roman type for rejected taxa. Numbers in parentheses correspond to notes. Synonyms used in references for Andalusian coasts are also presented. New unpublished records are shown by asterisks $(*)$ corresponding to a paper edited in this same volume (Conde et al., 1996).

Taxa are referenced by the first citation in the literature, except when a catalogue paper was published afterward. In this case, taxa are referenced by the cataloguing work. The references used to make the check-list were (numbers correspond to those shown in the check-list): 1: Agardh (1823); 2: Agardh (1824); 3: Agardh (1828); 4: Amo \& Mora (1870); 5: Ardré (1970); 6: Börgesen (1929); 7: Bornet (1892); 8: Clemente (1807); 9: Colmeiro (1867); 10: Colmeiro (1889); 11: 
Conde (1984); 12: Conde (1992); 13: Conde et al. (1990); 14: Conde \& Seoane (1982); 15: Conde \& Soto (1986); 16: Conde \& Soto (1987); 17: Cremades \& Pérez-Cirera (1990); 18: Dixon \& Irvine (1977); 19: Feldmann (1939); 20: Feldmann (1942); 21: Fernández et al. (1983); 22: Flores-Moya et al. (1994); 23: Flores-Moya et al. (1989); 24: Giaccone (1972); 25: González-Fragoso (1886); 26: GonzálezFragoso (1893); 27: González-Guerrero (1955); 28: Lemoine (1915); 29: Navarro \& Gallardo (1995); 30: Niell (1978); 31: Petersen (1918); 32: Rull et al. (1991); 33: Seoane (1965); 34: Soto (1991); 35: Soto \& Conde, (1989); 36: Toni \& Forti (1914); 37: Varo et al. (1979) and 38: Montagne in Webb (1853).

The taxonomic criteria from Conde (1991) are followed for Acrochaetiales. Ceramiales are arranged in accordance with Maggs \& Hommersand (1993). Corallinales and Hildenbrandiales are listed according to Irvine \& Chamberlain (1994), and Gigartinales according to Kraft \& Robins (1985). The criteria of Irvine (1983) are followed for Rhodymeniales. Bangiales, Bonnemaisoniales, Compsopogonales, Gelidiales, Nemaliales and Porphyridiales are listed according to Woelkerling (1990), and Gracilariales according to Fredericq \& Hommersand (1989).

Orders, families, genus and species are arranged alphabetically. Orders are labelled with Roman numbers corresponding to those shown in the alphabetical list of genra at end of the work, to permit an easier search for taxa of interest.

\section{LIST OF TAXA}

HU CA MA GR AL

\section{RHODOPHYCEAE Rabenhorst}

I. ACROCHAETIALES Feldmann emend. Garbary et Gabrielson

Acrochaetiaceae Fritsch ex W.R. Taylor

Audouiniella Bory

A. daviesii (Dillwyn)Woelkerling

A. endozoica (Darbishire) Dixon

A. leptonema (Rosenvinge) Garbary

A. microscopica (Nägeli) Woelkerling

A. moniliformis (Rosenvinge) Garbary

A. nemalionis (De Notaris) Dixon

A. parvula (Kylin) Dixon

A. saviana (Meneghini) Woelkerling

A. secundata (Lyngbye) Dixon

A. subpinnata (Bornet) Garbary

A. thuretii (Bornet) Woelkerling

A. trifila (Buffham) Dixon

A. virgatula (Harvey) Dixon

$\begin{array}{lllll}29 & - & - & 16 & 35 \\ - & - & - & 15 & - \\ - & - & - & - & 35 \\ 29 & - & - & - & 35 \\ - & - & - & - & 35 \\ - & - & - & 15 & 35 \\ 29 & - & - & 34 & 35 \\ - & - & - & - & 35 \\ - & - & - & - & 35 \\ - & - & - & - & 35 \\ 22 & - & - & 15 & - \\ - & - & 11 & 34 & 35 \\ - & - & - & 15 & 35\end{array}$


$\mathrm{HU}$ CA MA GR AL

II. BANGIALES F. Schmitz in Engler

\section{Bangiaceae Engler}

Bangia Lyngbye

B. atropurpurea (Roth) C. Agardh

$=\mathrm{B}$. fuscopurpurea var. atropurpurea Lyngbye

Porphyra C. Agardh

$P$. leucosticta Thuret in Le Jolis

P. umbilicalis (Linnaeus) J. Agardh

Porphyropsis Rosenvinge

P. coccinea (J. Agardh) Rosenvinge

III. BONNEMAISONIALES J. Feldmann et G. Feldmann

Bonnemaisoniaceae F. Schmitz in Engler

Asparagopsis Montagne

A. armata Harvey (1)

Bonnemaisonia (Woodward) C. Agardh

B. asparagoides (Woodward) C. Agardh

B. hamifera Hariot $^{(2)}$

Trailliella Batters

T. intricata Batters ${ }^{(3)}$

IV. CERAMIALES Oltmanns

Ceramiaceae Dumortier

Aglaothamnion Feldmann-Mazoyer ${ }^{(4)}$

A. byssoides (Arnot ex Harvey) L'Hardy-Halos et Rueness

$=$ Callithamnion byssoides Arnott ex Harvey

A. gallicum (Nägeli) Halos ex Ardré

A. hookeri (Dillwyn) Maggs et Hommersand

= Callithamnion hookeri (Dillwyn) S. F. Gray

A. tripinnatum (Grateloup ex C. Agardh) Mazoyer

= Callithamnion tripinnatum C. Agardh

Anotrichum Nägeli

A. barbatum (C. Agardh) Nägeli

A. furcellatum (J. Agardh) Baldock 
Antithamnion Nägeli

A. antillanum Boergesen

A. cruciatum (C. Agardh) Nägeli

A. tenuissimun (Hauck) Schiffner

HU CA MA GR AL

Antithamnionella Lyle

A. elegans (Berthold) Boudouresque et Verlaque

29

$\begin{array}{llll}- & - & - & -\end{array}$

$\begin{array}{llll}29 & 25 \quad-\quad 16 & 35\end{array}$

$29 \quad-\quad-\quad 16 \quad 35$

$=$ Antithamnion elegans Berthold

A. spirographidis (Schiffner) Wollaston

29

Bornetia Thuret

B. secundiflora (J. Agardh) Thuret

= Griffithsia secundiflora J. Agardh

Callithamniella Mazoyer

C. tingitana (Schousboe ex Bornet) G. Feldmann

Callithamnion Lyngbye

C. corymbosum (Smith) Lyngbye

C. granulatum (Ducluzeau) C. Agardh

C. neglectum (G. Feldmann) Ballesteros et Romero

C. tetragonum (Withering) S. F. Gray

C. tetricum (Dillwyn) S. F. Gray

Centroceras Kützing

C. clavulatum Montagne

33

11

35

Ceramium Roth

C. callipterum Mazoyer $^{(5)}$

C. ciliatum (J. Ellis) Ducluzeau

var.robustum (J. Agardh) Mazoyer

C. cingulatum Weber van Bosse

C. circinatum (Kützing) J. Agardh

C. codii (Richards) Mazoyer

C. diaphanum (Lightfoot) Roth var. lophophorum Mazoyer

var. zostericola Thuret

f. acrocarpum Mazoyer

C. echionotum J. Agardh

var. mediterraneum Mazoyer

C. flaccidum (Kützing) Ardissone

$=\mathrm{C}$. gracillimum Griffith et Harvey

var. byssoideum (Harvey) Mazoyer

C. gaditanum (Clemente) Cremades

$=\mathrm{C}$. flabelligerum J. Agardh

C. nodosum (Kützing) Harvey

$\begin{array}{lllll}- & 5 & - & - & - \\ 29 & 33 & 11 & 16 & 35 \\ - & 33 & 11 & 16 & 35 \\ - & * & - & - & 35 \\ - & - & - & - & 35 \\ - & - & - & 16 & 35 \\ 13 & 33 & 11 & 37 & 35 \\ 22 & - & - & - & - \\ - & - & 11 & 16 & 35 \\ - & - & - & - & 35 \\ - & 33 & - & 37 & 35 \\ - & - & 11 & 16 & - \\ 29 & 33 & 11 & 37 & 35 \\ & & & & \\ - & - & 11 & 16 & - \\ - & 33 & - & 37 & 35 \\ - & - & 11 & - & 35\end{array}$

$=$ C. tenuissimum (Roth) J. Agardh 
C. nodulosum (Lightfoot) Ducluzeau

= C. rubrum (Hudson) C. Agardh

var. barbatum (Kützing) J. Agardh

var. implexo-contortum Solier

var. tenue C. Agardh

C. pallidum (Nägeli ex Kützing) Maggs et Hommersand

C. strictum Harvey

$=$ C. diaphanum var. strictum Kützing

C. shuttlewortianum (Kützing) Silva ${ }^{(6)}$

$=\mathrm{C}$. acanthonotum Carmichael

C. taylorii Dawson

C. tenerrinum (Martens) Okamura

Compsothamnion Nägeli

C. thuyoides (Smith) F. Schmitz

$=$ Callithamnion thuyoides $\mathrm{C}$. Agardh

Crouania J. Agardh

C. attenuata (C. Agardh) J. Agardh

f. bispora (Crouan) Hauck

Griffithsia C. Agardh

G. corallinoides (Linnaeus) Batters

$=$ Conferva corallina Linnaeus

G. opuntioides J. Agardh

G. phyllamphora J. Agardh

G. schousboei Montagne ${ }^{(8)}$

Gymnothaminion J. Agardh

G. elegans (Schouboe ex C. Agardh) J. Agardh

Halurus Kützing

H. equisetifolius (Lightfoot) Kützing

H. flosculosus (Ellis) Maggs et Hommersand

= Griffithsia flosculosa (Ellis) Batters

= G. setacea (Hudson) C. Agardh

Lejolisia Bornet

L. mediterranea Bornet

Mesothamnion Boergesen

M. caribaeum Boergesen

Microcladia Greville

M. glandulosa (Solander ex Turner) Greville

= Delesseria glandulosa C. Agardh
$\mathrm{HU}$ CA MA GR AL

$\begin{array}{lllll}13 & 33 & 11 & 16 & 35\end{array}$

$\begin{array}{lllll}- & - & 11 & 16 & 35 \\ 22 & - & 11 & - & - \\ - & - & 11 & 16 & 35 \\ - & * & - & - & - \\ - & - & 11 & 16 & 35\end{array}$

26

29

$\begin{array}{lll}33 & 11 & 16\end{array}$

$25 \quad 11$ 
Monosporus Solier in Castagne

M. pedicellatus (Smith) Solier in Castagne var. tenuis Mazoyer

HU CA MA GR AL

Plenosporium Nägeli

$P$. borreri (Smith) Nägeli ex Hauck

= Callithamnion borreri C. Agardh

Pseudocrouania Funk

P. ischiana Funk

Pterothamnion Nägeli

$P$. crispum (Ducluzeau) Nägeli

P. plumula (J. Ellis) Nägeli

29

33

11

$37 \quad 35$

$=$ Anthithamnion plumula (Ellis) Thuret

= Platythamnion plumula•(Ellis) Boudouresque et al.

var. bebbii (Reinsch) Mazoyer

\section{Ptilothamnium}

P. pluma (Dillwyn) Thuret in Le Jolis

Seirospora Harvey

S. giraudyi (Kützing) De Toni

S. interrupta (Smith) F. Schmitz

S. sphaerospora J. Feldmann

Spermothamnion Areschoug

S. flabellatum Bornet

S. irregulare (J. Agardh) Ardissone

S. repens (Dillwyn) Rosenvinge

$=$ Callithamniom variabile C. Agardh

Sphondylothamnion Nägeli

S. multifidum (Hudson) Nägeli

= Griffithsia multifida C. Agardh

Spyridia Harvey in W. J. Hooker

S. aculeata (Schimper) Kützing

S. filamentosa (Wulfen) Harvey in Hooker

Tiffaniella Doty et Meñez

T. capitata (Schousboe) Doty et Meñez

= Spermothamnion capitatum (Schousboe) Bornet

Vickersia Karsakoff

V. baccata (J. Agardh) Karsakoff 
Wrangelia C. Agardh

W. penicillata C. Agardh

Dasyaceae Kützing

Dasya C. Agardh

D. corymbifera J. Agardh

D. elegans C. Agardh

D. hutchinsiae Harvey in Hooker

= D. arbuscula Harvey

D. ocellata (Grateloup) Harvey in Hooker

D. punicea Meneghini in Zanardini

D. rigidula (Kützing) Ardissone

Dasyopsis Zanardini

D. cervicornis (J. Agardh) F. Schmitz

D. spinella (C. Agardh) Zanardini $=$ Hutchinsia spinella C. Agardh

Heterosiphonia Montagne

H. crispella (C. Agardh) Wynne = Callithamnion crispellum C. Agardh

H. plumosa (J. Ellis) Batters = Dasya coccinea $\mathrm{C}$. Agardh

\section{Delesseriaceae Bory}

Acrosorium Zanardini ex Kützing

A. venulosum (Zanardini) Kylin

= A. reptans (Crouan) Kylin

= A. uncinatum (J. Agardh) Kylin

Apoglossun (J. Agardh) J. Agardh

A. ruscifolium (Turner) J. Agardh

Cottoniella Boergesen

C. filamentosa (M. Howe) Boergesen

Cryptopleura Kützing

C. ramosa (Hudson) Kylin ex L. Newton

= C. lacerata (S. G. Gmelin) Kützing

Drachiella Ernst et J. Feldmann

D. minuta (Kylin) Maggs et Hommersand

$$
=\text { Myriogramme minuta Kylin }
$$

Haraldiophyllum Zinova

H. bonnemaisonii (Kylin) Zinova 
Hypoglossum Kützing

H. hypoglosoides (Stackhouse) Collins et Hervey

HU CA MA GR AL $=\mathrm{H}$. woodwardii Kützing

Myriogramme Kylin

M. tristromatica (Rodriguez ex Mazza) Boudouresque

Nitophyllum Greville

N. flabellatum Ercegovic

N. punctatum (Stackhouse) Greville

Phycodrys Kützing

$P$. rubens (Linnaeus) Batters

$=\mathrm{P}$. sinuosa (Goodenough et Woodward) Kützing

Platysiphonia Boergesen

$P$. delicata (Clemente) Cremades ${ }^{(9)}$

Polyneura (J. Agardh) Kylin

P. bonnemaisonii (C. Agardh) Maggs et Hommersand ${ }^{(10)}$ $=$ P. hilliae (Greville) Kylin

Radicilingua Papenfuss

R. reptans (Kylin) Papenfuss

Taenioma J. Agardh

T. nanum (Kützing) Papenfuss

$=\mathrm{T}$. macruorum Thuret

Rhodomelaceae J. Areschoug

Alsidium C. Agardh

A. corallinum C. Agardh

A. helminthochortum (La Tourrette) Kützing

Aphanocladia Falkenberg

A. stichidiosa (Funk) Ardré

Boergeseniella Kylin

B. fruticulosa (Wulfen) Kylin

= Polysiphonia fruticulosa (Wulfen) Sprengel

B. thuyoides (Harvey in McKay) Kylin

$\begin{array}{llll}33 & 11 & 16 & 35 \\ 33 & 11 & 16 & 35\end{array}$

$=$ Polysiphonia thuyoides Harvey

= Pterosiphonia thuyoides (Harvey in McKay) F. Schmitz

= Rytiphloea thuyoides Harvey

Bostrychia Montagne

B. scorpioides (Hudson) Montagne 
Brongniartella Bory

B. byssoides (Goodenough et Woodward) F. Schmitz

HU CA MA GR AL

Chondria C. Agardh

C. boryana (De Notaris) De Tony

C. capillaris (Hudson) Wynne

$=\mathrm{C}$. tenuissima (Goodenough et Woodward) J. Agardh

C. coerulescens (J. Agardh) Falkenberg

C. dasyphylla (Woodward) C. Agardh

C. mairei J. Feldmann

C. scintillans G. Feldmann

Ctenosiphonia Falkenberg

C. hypnoides (Welwitsch ex J. Agardh) Falkenberg

Digenea $\mathrm{C}$. Agardh

D. simplex (Wulfen) C. Agardh

Dipterosiphonia F. Schmitz et Falkenberg

D. dendritica (C. Agardh) F. Schmitz in Engler et Prantl

D. rigens (Schousboe) Falkenberg

Erytrocystis J. Agardh

E. montagnei (Derbès et Solier) Silva

$\begin{array}{lllll}29 & 33 & 11 & 37 & - \\ - & 1 & 11 & - & 35 \\ - & - & - & - & 35 \\ - & - & - & - & 35\end{array}$

Halopithys Kützing

$H$. incurvus (Hudson) Batters

$=H$. pinastroides $(\mathrm{S} . \mathrm{G}$. Gmelin) Kützing

Herposiphonia Nägeli

H. secunda (C. Agardh) Ambronn

f. tenella (C. Agardh) Wynne

$=$ H. tenella (C. Agardh) Nägeli

$\begin{array}{lllll}29 & 33 & 11 & 16 & 35\end{array}$

$\begin{array}{lllll}29 & 33 & 11 & 16 & 35\end{array}$

Laurencia Lamouroux

L. hybrida (A.L.P.P. de Candolle) Lenormand ex Duby = L. caespitosa Lamouroux

L. microcladia Kützing

L. obtusa (Hudson) Lamouroux

L. paniculata (C. Agardh) J. Agardh

L. papillosa (Foorsskal) Greville $=$ Fucus tenerrimus Clemente

L. pinnatifida (Hudson) Lamouroux

L. pyramidalis Bory ex Kützing = L. obtusa var. pyramidata J. Agardh 
Lophosiphonia Falkenberg

L. cristata Falkenberg

L. reptabunda (Suhr in Kützing) Kylin

L. scopulorum (Harvey) Womersley

L. subadunca (Kützing) Falkenberg

$\mathrm{HU}$ CA MA GR AL

Ophidocladus Falkenberg

O. simpliciusculus (Crouan frat.) Falkenberg

= Polysiphonia simpliciuscula Crouan

Osmundaria Lamouroux

O. volubilis (Linnaeus) Norris

= Vidalia volubilis (Linnaeus) J. Agardh

Polysiphonia Greville

P. atra Zanardini

$P$. atlantica Kapraun et Norris

= P. macrocarpa Harvey in Mackay

$P$. biformis Zanardini

P. breviarticulata (C. Agardh) Zanardini

P. brodiaei (Dillwyn) Sprengel

$=$ Hutchinsia brodiaei C. Agardh

P. cladorrhiza Ardissone

$P$. deludens Falkenberg

$P$. denudata (Dillwyn) Greville ex Harvey in Hooker

$P$. dichotoma Kützing

P. elongata (Hudson) Sprengel

= Hutchinsia elongata C. Agardh

P. erythraea (Schousboe) J. Agardh ${ }^{(12)}$

$P$. fibrillosa (Dillwyn) Sprengel

$=$ P. spinulosa Greville

P. flexella (C. Agardh) J. Agardh

$=$ Hutchinsia flexella C. Agardh

P. flocculosa (C. Agardh) Kützing

$=$ Hutchinsia subcontinua C. Agardh

P. fucoides (Hudson) Greville

$\begin{array}{lllll}- & - & - & - & 35 \\ - & 33 & 11 & 16 & 35 \\ - & - & - & - & 35\end{array}$

$29 \quad-\quad-\quad-35$

$=$ P. nigrescens (Hudson) Greville in Hooker

$=\mathrm{P}$. violacea $($ Roth $)$ Sprengel

= Hutchinsia nigrescens C. Agardh

P. furcellata (C. Agardh) Harvey in Hooker

P. hirta J. Agardh

= Hutchinsia flocculosa C. Agardh var. fibrillosa C. Agardh

$P$. lanosa (Linnaeus) Tandy

$=$ P. fastigiata Grevile

P. obscura (C. Agardh) J. Agardh ${ }^{(14)}$

$=$ Hutchinsia obscura C. Agardh

P. opaca (C. Agardh) Moris et De Notaris

P. polyspora (C. Agardh) J. Agardh ${ }^{(15)}$ 


\begin{tabular}{|c|c|c|c|c|}
\hline & $\mathrm{HU}$ & $\mathrm{CA}$ & MA & GR \\
\hline P. ruchingeri J. Agardh & - & - & - & 15 \\
\hline $\begin{array}{l}\text { P. sanguinea (C. Agardh) Zanardini }{ }^{(16)} \\
\quad=\text { P. fibrata (Dillwyn) Harvey in Hooker }\end{array}$ & - & - & 23 & - \\
\hline P. scopulorum Harvey & 29 & - & - & - \\
\hline P. sertularoides (Grateloup) J. Agardh & - & 7 & 11 & - \\
\hline P. simulans Harvey ${ }^{(17)}$ & - & 7 & - & - \\
\hline P. stuposa Zanardini & - & - & - & 15 \\
\hline P. subulata (Dillwyn) J. Agardh & - & - & 11 & - \\
\hline P. subulifera (C. Agardh) Harvey & - & - & 11 & - \\
\hline P. tenerrima Kützing & - & - & - & 16 \\
\hline P. tripinnata J. Agardh & - & - & - & 15 \\
\hline rosiphonia Falkenberg in F. Schmitz et Falkenberg & & & & \\
\hline P. complanata (Clemente) Falkenberg & - & 33 & 11 & 16 \\
\hline P. parasitica (Hudson) Falkenberg & - & 33 & 11 & - \\
\hline $\begin{array}{l}\text { P. pennata }(\mathrm{C} \text {. Agardh) Falkenberg } \\
\quad=\text { Polysiphonia pennata (Roth) Falkenberg }\end{array}$ & 13 & 33 & 11 & 37 \\
\hline
\end{tabular}

Rytiphloea C. Agardh

R. tinctoria (Clemente) C. Agardh

\section{COMPSOPOGONALES Skuja}

\section{Erythropeltidaceae Skuja}

Erythrotrichia J. Areschoug

E. carnea (Dillwyn) J. Agardh

= E. reflexa (H. M. et P. L. Crouan) Thuret ex De Toni

\section{Shalingia Kornmann}

S. subintegra (Rosenvinge) Kornmann

= Erythrocladia subintegra Rosenvinge

$=$ E. irregularis Rosenvinge

= Erythrochloropeltis subintegra (Rosenvinge) Kornmann et Sahling

= Erythropeltis subintegra (Rosenvinge) Kornmann et Sahling

\section{CORALLINALES Silva et Johansen}

Corallinaceae Lamouroux

Amphiroa Lamouroux
A. beauvoisii Lamouroux
A. cryptarthrodia Zanardini
A. rigida Lamouroux

$\begin{array}{lllll}- & 33 & 11 & 16 & 35 \\ - & - & - & 16 & 35 \\ - & 33 & 11 & - & 35\end{array}$


$\mathrm{HU} \quad \mathrm{CA} \quad \mathrm{MA} \quad \mathrm{GR} \quad \mathrm{AL}$

Choreonema F. Schmitz emend. Woelkerling

C. thuretii (Bornet) F .Schmitz

$16 \quad 35$

Corallina Linnnaeus

C. elongata J. Ellis et Solander

= C. mediterranea J. Areschoug

C. granifera J. Ellis et Solander

C. officinalis Linnaeus

Fosliella Howe

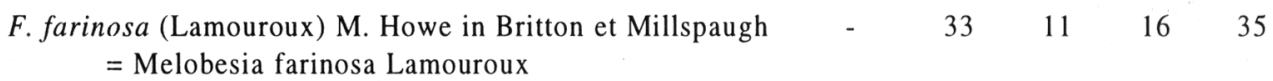
var. solmsiana (Falkenberg) Foslie

Goniolithon Foslie

G. papillosum (Zanardini ex Hauck) Foslie

Jania Lamouroux

$J$. adhaerens Lamouroux

J. longifurca Zanardini

$J$. rubens (Linnaeus) Lamouroux

var. corniculata (Linnaeus) Yendo

$\begin{array}{llll}33 & 11 & 16 & 35\end{array}$

$\begin{array}{llll}33 & 11 & - & 35\end{array}$

$\begin{array}{llll}33 & 11 & 16 & 35\end{array}$

$=\mathrm{J}$. corniculata (Linnaeus) Lamouroux

Lithophyllum Philippi

L. expansum Philippi

L. incrustans Philippi

f. depressa Foslie

f. flabellata Heydrich

L. lichenoides Philippi ${ }^{(18)}$

= L. tortuosum auct. sensu Lemoine

$=$ Tenarea tortuosa (Esper) Lemoine

Lithothamnion Heydrich

L. valens Foslie

Melobesia Lamouroux

M. membranacea (Esper) Lamouroux

= Epilithon membranaceum (Esper) Heydrich

= Lithothamnion membranaceum Foslie

Mesophyllum Lemoine

M. lichenoides (J. Ellis et Solander) Lemoine

Phymatolithon Foslie emend W. Adey

P. calcareum (Pallas) W. Adey et McKibbin

P. lenormandii (J. Areschoug) W. Adey

$\begin{array}{lll}11 & 34 & - \\ - & 16 & 35\end{array}$


Pneophyllum Kützing

HU CA MA GR AL

$P$. confervicola (Kützing) Chamberlain

= Fosliella minutula (Foslie) Ganesan

35

$P$. lejolissii (Rosanoff) Chamberlain

P. caulerpae (Foslie) P. Jones et Wolkerling

$=\mathrm{P}$. zonale (Crouan frat.) Chamberlain

Spongites Kützing

S. dentata Kützing

= Lithophyllum dentatum (Kützing) Foslie

$\begin{array}{cccc}13 & 15 & 16 & 35 \\ - & - & 34 & - \\ - & - & - & 35\end{array}$

S. notarisii (L. Dufour) Athanasiadis

= Melobesia notarisii L. Dufour

$=$ Neogoniolithon notarisii (L. Dufour) Setchell et Mason

S. ramulosa (Philippi) Kützing

Titanoderma Nägeli

T. corallinae (P. et H. Crouan) Woelkerling et al.

$=$ Dermatolithon corallinae (Crouan) Foslie

T. cystoseirae (Hauk) Woelkerling et al.

T. hapalidioides (P. et H. Crouan) J. Price et al.

T. litorale (P. et H. Crouan) Boudouresque et Perret

$=$ Dermatolithon litorale (Suneson) Lemoine

T. mediterranea (Foslie) Woelkerling

= Litholepis mediterranea Foslie

T. pustulatum (Lamouroux) Nägeli

$=$ Dermatolithon pustulatum (Lamouroux) Foslie

$=$ Lithophyllum pustulatum Foslie

VII. GELIDIALES Kylin

\section{Gelidiaceae Kützing}

Gelidium Lamouroux

G. latifolium (Greville) Bornet et Thuret (20)

var. histrix (J. Agardh) Hauck

G. melanoideum Schousboe ex Bornet var. gracile J. Feldmann et Hamel

G. pectinatum Schousboe ex Montagne

G. pusillum (Stackhouse) Le Jolis (21) var. pulvinatum (C. Agardh) J. Feldmann

G. sesquipedale (Clemente) Thuret in Bornet et Thuret

G. spathulatum (Kützing) Bornet

G. spinulosum (C. Agardh) J. Agardh

$\begin{array}{lllll}29 & 33 & 11 & 16 & 35 \\ - & - & - & - & 35 \\ - & - & - & - & 35 \\ - & 33 & - & - & - \\ - & 19 & 11 & 34 & - \\ 13 & 33 & 11 & 16 & 35 \\ - & 33 & - & 37 & - \\ - & 33 & 11 & - & - \\ 22 & 33 & - & - & - \\ - & 33 & 11 & - & 35\end{array}$

$=\mathrm{G}$. microdon Kützing

Pterocladia J. Agardh

P. capillacea (S. G. Gmelin) Bornet et Thuret 
HU CA MA GR AL

\section{Gelidiellaceae Fan}

Gelidiella J. Feldmann et Hamel

G. lubrica (Kützing) J. Feldmann et Hamel

G. nigrescens (J. Feldmann) J. Feldmann et Hamel

G. pannosa (J. Feldmann) J. Feldmann et Hamel

$\begin{array}{lllll}- & - & - & 34 & 35 \\ - & * & - & 15 & - \\ - & - & - & - & 35\end{array}$

VIII. GIGARTINALES Schmitz in Engler

\section{Caulacanthaceae Kützing}

Catenella Greville

C. caespitosa (Withering) L. Irvine in Parke et Dixon $=$ C. opuntia Greville

$=$ C. repens Batters

Caulacanthus Kützing

C. ustulatus (Mertens) Kützing (22)

$\begin{array}{lllll}29 & 33 & 11 & 16 & 35\end{array}$

Choreocolacaceae Sturch

Gelidiocolax Gardner

G. cf christianae J. Feldmann et G. Mazoyer

Cystocloniaceae Kützing

\section{Calliblepharis Kützing}

C. ciliata (Hudson) Kützing

$=$ Fucus ciliatus Linnaeus

C. jubata (Goodenoug et Woodward) Kützing

= Fucus ciliatus var. jubatus Turner

Cystoclonium Kützing

C. purpureum (Hudson) Batters ${ }^{(23)}$

$=\mathrm{C}$. purpurascens Kützing

Rhodophyllis Kützing

$R$. divaricata (Stackhouse) Papenfuss

$1 \quad 11,22$

35

= Sphaerococcus bifidus C. Agardh

Furcellariaceae Greville

Furcelleria Lamouroux

F. lumbricalis (Hudson) Lamouroux ${ }^{(24)}$

= F. fastigiata Lamouroux 
HU CA MA GR AL

Gigartinaceae Bory

Gigartina Stackhouse

G. acicularis (Roth) Lamouroux

G. pistillata (S. G. Gmelin) Stackhouse

G. teedii (Roth) Lamouroux

$\begin{array}{lllll}29 & 33 & 11 & 16 & 35 \\ - & 33 & 23 & - & - \\ - & 33 & 11 & 37 & -\end{array}$

Mastocarpus Kützing

M. stellatus (Stackhouse in Withering) Guiry in Guiry et al. - $\quad 1^{(25)}$ = Sphaerococcus mamillosus C. Agardh

Gloiosiphoniaceae F. Schmitz

Thuretella F. Schmitz in Engler et Prantl

T. schousboei (Thuret) F. Schmitz in Engler et Prantl (26)

\section{Gymnophlaeaceae Kützing}

Platoma F. Schmitz

P. incrassata Schousboe (27)

Schizymenia J. Agardh

S. dubyi (Chauvin ex Duby) J. Agardh

$11 \quad 37$

\section{Halymeniaceae Bory}

Acrodiscus Zanardini

A. vidovichii (Meneghini) Zanardini

Cryptonemia J. Agardh

C. seminervis (C. Agardh) J. Agardh

$\begin{array}{llll}33 & 11 & 37 & 35\end{array}$

= C. lomation (Berthold) J. Agardh

Grateloupia C. Agardh

G. doryphora (Montagne) Howe

G. filicina (Lamouroux) C. Agardh

G. lanceola (J. Agardh) J. Agardh ${ }^{(28)}$

Halymenia C. Agardh

H. floresia (Clemente) C. Agardh (29)

H. latifolia $\mathrm{P}$. et $\mathrm{H}$. Crouan

$\begin{array}{ccccc}29^{-} & 33 & - & - & 35 \\ - & - & 11 & - & -\end{array}$

Hypneaceae J. Agardh

Hypnea Lamouroux

H. cervicornis J. Agardh

H. coccinea (Clemente) Cremades

= Fucus plicatus var. coccineus Clemente

H. musciformis (Wulfen) Lamouroux

$\begin{array}{lllll}29 & 33 & 11 & - & 35\end{array}$


$\mathrm{HU}$ CA MA GR AL

Kallymeniaceae W. R. Taylor

Callophyllis Kützing

C. laciniata (Hudson) Kützing

$=$ Fucus laciniatus Hudson

Kallymenia J. Agardh
K. microphylla J. Agardh (30)
K. reniformis (Turner) J. Agardh
K. spathulata (J. Agardh) Codomier

\section{Peyssomneliaceae Denizot}

Peyssonnelia Decaisne

$P$. armorica (P. et H. Crouan) Boergesen

$P$. atropurpurea $\mathrm{P}$. et $\mathrm{H}$. Crouan

$P$. bornetii Boudouresque et Denizot

$P$. coriacea J. Feldmann

$P$. dubyi P. et H. Crouan

$P$. harveyana $\mathrm{P}$. et $\mathrm{H}$. Crouan in J. Agardh

$P$. inamoena $\mathrm{Pilger}$

$P$. polymorfa (Zanardini) F. Schmitz in Falkenberg

$P$. rosa-marina Boudouresque et Denizot

P. rubra (Greville) J. Agardh

P. squamaria (S. G. Gmelin) Decaisne = Fucus squamarius S. G. Gmelin

P. stoechas Boudouresque et Denizot

Polystrata Heydrich

P. compacta (Foslie) Denizot

P. fosliei (Weber van Bosse) Denizot

\section{Phyllophoraceae Nägeli}

Gymnogongrus G. Martius
G. crenulatus (Turner) J. Agardh (31)
$=\mathrm{G}$. norvegicus J. Agardh
G. devoniensis (Greville) Schotter (3!)
G. griffithsiae (Turner) G. Martius
G. patens J. Agardh

$\begin{array}{lllll}29 & 33 & 11 & - & - \\ - & 21 & 21 & - & - \\ 29 & 33 & 11 & 37 & 35 \\ - & 33 & - & 37 & -\end{array}$

Phyllophora Greville
P. crispa (Hudson) Dixon $=\mathrm{P}$. rubens (Goodenough et Woodward) Greville = P. epiphylla (Müller) Batters

$P$. heredia (Clemente) J. Agardh 
HU CA MA GR AL

$P$. sicula (Kützing) Guiry et L. Irvine

P. truncata (Pallas) A. D. Zinova

Schottera Guiry et Hollenberg

S. nicaensis (Lamouroux ex Duby) Guiry et Hollenberg

$\begin{array}{ccccc} & & & & \\ - & - & 11 & - & - \\ - & - & 11 & - & - \\ - & * & 11 & 16 & 35 \\ - & 38 & - & - & -\end{array}$

\section{Plocamiaceae Kützing}

Plocamium Lamouroux

P. cartilagineum (Linnaeus) Dixon $=\mathrm{P}$. coccineum (Hudson) Lyngbye

P. raphelisianum (J. Agardh) P. A. Dangeard ${ }^{(33)}$

$\begin{array}{lllll}22 & 33 & 11 & 16 & 35\end{array}$

Polyidaceae Kylin

Polyides C. Agardh

$P$. rotundus (Hudson) Greville = Fucus rotundus S.G. Gmelin

$29 \quad 8 \quad 11^{(34)}$

Rhizophyllidaceae F. Schmitz

Contarinia Zanardini

C. squamariae (Meneghini) Denizot

Rissoellaceae Kylin

Rissoella J. Agardh

$R$. verruculosa (Bertholdi) J. Agardh

$11 \quad 16$

Solieriaceae (Harvey) Hauck

Solieria J. Agardh

S. chordalis (C. Agardh) J. Agardh (35)

$=$ Delesseria chordalis C. Agardh

Sphaerococcaceae Dumortier

Sphaerococcus Stackhouse

S. coronopifolius Stackhouse

$\begin{array}{llll}33 & 11 & 16 & 35\end{array}$

Wurdemanniaceae W. R. Taylor

Wurdemannia Harvey

W. miniata (Manoury) J. Feldmann et Hamel 
IX. GRACILARIALES Fredericq et Hommersand

$\mathrm{HU}$ CA MA GR AL

Gracilariaceae (Nägeli) Kylin

Gracilaria Greville

G. armata (C. Agardh) J. Agardh

G. bursa-pastoris (S. G. Gmelin) Silva

- $611 \quad-\quad 35$

$=\mathrm{G}$. compressa C. Agardh

$\begin{array}{lllll}29 & 26 & 11 & -\end{array}$

G. cervicornis (Turner) J. Agardh

G. dura (C. Agardh) J. Agardh

$=$ Sphaerococcus durus C. Agardh

G. multipartita (Clemente) Harvey ${ }^{(37)}$

$=\mathrm{G}$. foliifera (Forsskal) Boergesen

G. verrucosa (Hudson) Papenfuss

$=$ Fucus confervoides Linnaeus

\section{HILDENBRANDIALES Pueschel et Cole}

Hildenbrandiaceae Rabenhorst

Hildenbrandia Nardo

H. canariensis Boergesen

H. rubra (Sommerfelt) Meneghini

$=\mathrm{H}$. prototypus Nardo

XI. NEMALIALES F. Schmitz in Engler

Chaetangiaceae Kützing

Scinaia Bivona

S. forcellata Bivona-Bernardi ${ }^{(38)}$

$13 \quad 33 \quad 11$

$=\mathrm{S}$. pseudocrispa (Clemente) Wynne

S. turgidla Chemin

Helminthocladiaceae J. Agardh

Helminthocladia J. Agardh

H. stackhousei (Clemente) Cremades ${ }^{(39)}$

$=$ Helminthora divaricata (C. Agardh) J. Agardh

$=$ H. stackhousei (Clemente) Cremades et Pérez-Cirera

Liagora Lamouroux

L. ceranoides Lamouroux

L. distenta (G. Mertens ex Roth) C. Agardh

L. farinosa Lamouroux

L. tetrasporifera Boergesen

L. viscida (Forsskal) C. Agardh 


\section{Naccariaceae Kylin}

HU CA MA GR AL

Atractophora Crouan frat.

A. hypnoides Crouan frat. ${ }^{(40)}$

Nemalionaceae (Farlow) De Toni et Levi

Nemalion Duby

$N$. helminthoides (Velley in Withering) Batters

$33 \quad 11 \quad 16$

35

XII. PORHYRIDIALES Kylin

Goniotrichaceae G. M. Smith

Chroodactylon Hansgirg

C. ornatum (C. Agardh) Basson

Stylonema Reinsch

S. alsidii (Zanardini) Drew

$\begin{array}{lllll}29 & 33 & 11 & 16 & 35\end{array}$

= Goniotrichum elegans (Chauvin) Le Jolis

$=\mathrm{G}$. alsidii (Zanardini) Howe

S. cornu-cervi Reinsch

= Goniotrichum cornu-cervi (Reinsch) Hauck

XIII. RHODYMENIALES F. Schmitz in Engler

Champiaceae Kützing

Champia Desvaux

C. parvula (C. Agardh) Harvey

$\begin{array}{lllll}13 & 33 & 11 & 16 & 35\end{array}$

Chylocladia Greville in Hooker

C. verticillata (Lightfoot) Bliding

= C. kaliformis (Goodenough et Woodward) Greville

$\begin{array}{lllll}13 & 33 & 11 & - & 35\end{array}$

Gastroclonium Kützing

G. clavatum (Roth) Ardissone

G. ovatum (Hudson) Papenfuss

$\begin{array}{lllll}22 & 33 & 23 & 16 & 35\end{array}$

$=$ Fucus ovalis Hudson

G. reflexum (Chauvin) Kützing

$29 \quad 4$

Lomentariaceae J. Agardh

Lomentaria Lyngbye

L. articulata (Hudson) Lyngbye

L. clavaeformis Ercegovic

L. clavellosa (Turner) Gaillon

= Gigartina clavellosa Lamouroux

$\begin{array}{lllll}- & 33 & 11 & 16 & - \\ - & - & 11 & - & - \\ 29 & 25 & 11 & - & -\end{array}$


L. compressa (Kützing) Kylin

L. ercegovicii Verlaque et al.

L. linearis Zanardini

L. verticillata Funk

\section{Rhodymeniaceae Harvey}

Botryocladia Kylin

$B$. botryoides (Wulfen) J. Feldmann

B. microphysa (Hauck) Kylin

Chrysimenia J. Agardh

C. ventricosa (Lamouroux) J. Agardh

= Halymenia ventricosa C. Agardh

Cordylecladia J. Agardh

C. erecta (Greville) J. Agardh

Fauchea Bory et Montagne

F. microspora Bornet in Rodriguez ${ }^{(42)}$

F. repens (C. Agardh) Montagne ${ }^{(43)}$

= Sphaerococcus repens C. Agardh.

Rhodymenia Greville

$R$. ardissonei J. Feldmann

$R$. pseudopalmata (Lamouroux) Silva

$=$ R. palmetta Greville

= Fucus bifidus Hudson

\section{NOTES}

Several records in papers prior to 40 years ago correspond to plants collected as drift, and not attached to any substratum. Thus, only the presence/ absence of tha taxa, rather than their identification will be discussed.

(1). Including the tetrasporophyte Falkenbergia rufolanosa (Harvey) F. Schmitz. See FloresMoya \& Conde (1992).

(2). Considered as taxon inquirenda. It needs confirmation for Málaga.

(3). The tetrasporophyte of Bonnemaisonia hamifera Hariot.

(4). See the acceptance of the genus Aglaothamnion in Maggs \& Hommersand (1993: 87).

(5). Considered as taxon inquirenda because it is the only citation for Andalusia. Colmeiro

$\begin{array}{lllll}\text { HU } & \text { CA } & \text { MA } & \text { GR } & \text { AL } \\ - & - & - & 15 & - \\ - & - & - & 34 & - \\ - & - & - & - & 35 \\ - & - & - & - & 35\end{array}$

35

$34 \quad-$

$\begin{array}{lllll}- & - & - & - & 35 \\ - & 25 & 11^{(44)} & 37 & -\end{array}$

(1867: 199) cited Ceramium cancellatum D.C. (sub Conferva cancellata Clem. Ens.) but Ardré (1970: 289) pointed out that it is possible that the record from Colmeiro could be this species.

(6). Ardré (1970: 282) proposed that the Tajo river (Portugal) is the southern geographical limit for this species. Because this is the only citation is therefore considered as taxon inquirenda for Andalusia.

(7). The first citation corresponds to Clemente (1807: 322) (sub Conferva corallina Linnaeus). Bellón (1942:73) pointed out that the specific epithet proposed by Linnaeus is «corallioides» and not «corallinoides».

(8). Considered as taxon inquirenda becasue is the only citation for Andalusia (Amo \& Mora, 1870:95). It could correspond to Griffithsia 
opuntioides J. Agardh.

(9). Taxon inquirenda because no new records have been reported since the first one from Clemente (1807).

(10). Needs confirmation because it has been collected only as drift.

(11). This species inhabits the SE Iberian Peninsula (Soto \& Conde: 1994). However, it has been considered as taxon inquirenda (see Silva, 1992).

(12). Taxon inquirenda. The only citation corresponds to Bornet (1892: 308). Feldmann (1981) included this species in the "Oligosiphonia spinulosae» group, with species not found in Andalusia.

(13). The reference from Agardh (1824:152) for Cádiz is the only one for Andalusia. However, it is included in a taxon (Breviarticulatae) with elements from the Mediterranean Sea (Feldmann, 1981:75).

(14). Taxon inquirenda. The only one citation correspond to Agardh (1828: 109) sub Hutchinsia obscura.

(15).Taxon inquirenda. The only citation corresponds to Agardh (1824:153).

(16). See the comments of Maggs \& Hommersand (1993: 333) in relation to Polysiphonia sanguinea C. Agardh.

(17). Taxon inquirenda. The only citation correspond to Bornet (1892: 315).

(18). Cabioch et al. (1992) considered Lithophyllum lichenoides Philippi and Tenarea tortuosa (Esper) Lemoine as different species.

(19). Giaccone (1972:43) recorded Neogoniolithon mamillosum (Hauck) Setchell \& Masson but Athanasiadis (1987: 39) pointed out that they are conspecific.

(20). This taxon includes Gelidium attenuatum (Turner) Thuret

(21). According to Dixon \& Irvine (1977: 311), this species includes Gelidium crinale (Turner) Lamouroux. and Gelidium pulchellum (Turner) Kützing.

(22). Mediterranean plants could correspond to Feldmannophycus rayssiae (J. Feldmann et G. Feldmann) Augier et Boudouresque (Augier \& Boudouresque, 1971).

(23). Taxon inquirenda.

(24). Could be misidentified as Polyides rotundus (Hudson) Greville. See Dixon \& Irvine (1977: 180). See note \#34.

(25). Has not been found later.

(26). This species occurs in the Mediterranean Sea but the records in Andalusia correspond to Cádiz (Atlantic Ocean) (Bornet, 1892: 336; Sauvageau, 1899: 698 and Feldmann, 1939:
327). It has been considered as taxon inquirenda.

(27). Has been found in Cádiz as drift. This species is close to Platoma marginifera (J. Agardh) Batters and it has a similar geographic distribution.

(28). A sheet from the Herbario de la Sociedad Malagueña de Ciencias (Conde, 1992: 37) annotated as Schizymenia dubyi, could be misidentified as Grateloupia lanceola. See Pérez-Cirera et al. (1989: 124, 131 and 133).

(29). See Silva (1992: 169) and Cremades (1993: 15) for taxonomic priority.

(30). Taxon inquirenda. Bornet (1892:278) recorded it from the herbarium Schousböe from Tangiers (Morocco).

(31). According to J. Feldmann in Schotter (1968: 47), the taxon could be a form from the synonym. However, see Dix on \& Irvine (1977: 217). Fernández et al. (1983: 164) separated Gymnogongrus crenulatus (Turner) J. Agardh and Gymnogongrusdevoniensis (Greville) Schotter.

(32). Found on the Atlantic coast, as was pointed out by Amo y Mora (1870: 183). However, the citations correspond to the 19 th century. Thus, it has been considered as taxon inquirenda.

(33). Needs to be studied in the Andalusian coasts.

(34). Taxon inquirenda for Málaga. Bellón (1942: 56) pointed out that Colmeiro $(1867,1889)$ cited this species as collected by Clemente. However, the locality is not specificated in their work "Ensayo ... Flora Boetica". On the other hand, it could be misidentified as Furcellaria lumbricalis (Hudson) Lamouroux (see note \#24).

(35). All citations correspond to 19 th century. Ardré (1970: 240) found this species in South Portugal as drift. Therefore, it has been considered as taxon inquirenda.

(36). Has not been found later.

(37). See Silva (1992) and Cremades (1993) for taxonomic priority.

(38). In accordance with Silva (1992: 167) the valid name is Sciania furcellata (Turner) J. Agardh.

(39). See Cremades (1993: 22) for the typification of the taxon.

(40). Taxon inquirenda. Seoane (1965: 96) records the only citation as drift.

(41). Taxon excludenda for Granada (Conde \& Soto, 1987: 37).

(42). Taxon inquirenda. All the records are from the 19th century. Sauvageau (1899: 698) reported this species in Cádiz, Tangiers and Western Mediterranean.

(43). Like note \#42. See also Ardissone (1883: 207).

(44). Bellón (1942: 67) commented that this species does not occur in the Mediterranean Sea and 
the possible misidentification with a few Phyllophoraceae (see also Irvine, 1983: 96). Taking into account the previous comment and the poor references, it has been considered as taxon inquirenda for Málaga.

\section{TAXA EXCLUDENDA}

Delesseria sanguinea (Hudson) Lamouroux. Cádiz, (González-Fragoso, 1886: 129, sub Delesseria sanguinea Lamouroux). Málaga, (Prolongo, 1852: 127, sub Fucus sanguineus Linnaeus). Ardré (1970: 317 ) reported that the southern limit for this species is North Portugal.

Dilsea carnosa (Schmidel) O. Kuntze. Cádiz, (González-Fragoso, 1886: 126, sub Iridaea edulis Bory). Málaga, (Agardh, 1823: 202 and 203, sub Halymenia edulis). Ardré (1970: 207) proposed that the southern limit for this species is the Duero river.

Dumontia contorta (S. G. Gmelin) Ruprecht. Cádiz, (González-Fragoso, 1886: 125, sub Dumontia filiformis Greville). Ardré (1970: 206) pointed out that the southern limit of this species is the Duero river.

Halimenia trigona (Clemente) C. Agardh. Cádiz, (Clemente, 1807: 318, sub Fucus trigonus). See Cremades (1993: 25).

Phyllophora pseudoceranoides (S. G. Gmelin) Newroth \& A. R. A. Taylor. Cádiz, (Clemente, 1807: 308, sub Fucus membranifolius Linnaeus). According to Ardré (1970: 251), it could correspond to Phyllophora sicula (Kützing) Guiry et L. Irvine.

Plumaria plumosa (Hudson) O. Kuntze. Cádiz, (Colmeiro, 1889: 931, sub Ptilota plumosa Agardh). Ardré (1970:293) pointed out that it is a mistake both from Clemente (1807: 318) and Colmeiro (1867: 200 y 1889: 930). The geographical southern limit of this species is North Portugal.

Rhodomela lycopodioides (L.) C. Agardh. Cádiz, (González-Fragoso, 1893: 162, sub Rhodomela lycopodioides Linnaeus). Maggs \& Hommersand (1993: 297) pointed out the possible misidentification as Rhodomela confervoides (Hudson) Silva.

\section{ALPHABETIC LIST OF GENRE}

Roman numbers corresponds to Orders from the List of Taxa, $\mathrm{N}$ to the Notes and TE to Taxa Exludenda chapters. Italics has been used for accepted genra and Roman type for rejected genra.

\begin{tabular}{|c|c|}
\hline Acrodiscus & VIII \\
\hline Acrosorium & IV \\
\hline Aglaothamnion & IV, N \\
\hline Alsidium & IV \\
\hline Amphiroa & VI \\
\hline Anotrichum & IV \\
\hline Anthithamnion & IV \\
\hline Antithamnion & IV \\
\hline Antithamnionella & IV \\
\hline Aphanocladia & IV \\
\hline Apoglossum & IV \\
\hline Asparagopsis & III \\
\hline Atractophora & XI \\
\hline Audouiniella & I \\
\hline Bangia & II \\
\hline Bangia & II \\
\hline Boergeseniella & IV \\
\hline Bonnemaisonia & III, N \\
\hline Bornetia & IV \\
\hline Bostrychia & IV \\
\hline Botryocladia & XIII \\
\hline Brongniartella & IV \\
\hline Calliblepharis & VIII \\
\hline Callithamniella & IV \\
\hline Callithamnion & IV \\
\hline Callithamnion & IV \\
\hline Callophyllis & VIII \\
\hline Catenella & VIII \\
\hline Catenella & VIII \\
\hline Caulacanthus & VIII \\
\hline Centroceras & IV \\
\hline Ceramium & IV, N \\
\hline Champia & XIII \\
\hline Chondria & IV \\
\hline Chondria & IV \\
\hline Choreonema & VI \\
\hline Chroodactylon & XII \\
\hline Chrysimenia & XIII \\
\hline Chylocladia & XIII \\
\hline Chylocladia & XIII \\
\hline Compsothamnion & IV \\
\hline
\end{tabular}




\begin{tabular}{|c|c|c|c|}
\hline Conferva & IV, N & Griffithsia & IV \\
\hline Contarinia & VIII & Gymnogongrus & VIII, N \\
\hline Corallina & VI & Gymnogongrus & VIII \\
\hline Cordylecladia & XIII & Gymnothamnion & IV \\
\hline Cottoniella & IV & Halopithys & IV \\
\hline Crouania & IV & Halopithys & IV \\
\hline Cryptonemia & VIII & Halurus & IV \\
\hline Cryptonemia & VIII & Halymenia & VIII \\
\hline Cryptopleura & IV & Halymenia & XIII, TE \\
\hline Ctenosiphonia & IV & Haraldiophyllum & IV \\
\hline Cystoclonium & VIII & Helminthocladia & XI \\
\hline Cystoclonium & VIII & Helminthora & XI \\
\hline Dasya & IV & Herposiphonia & IV \\
\hline Dasyopsis & IV & Herposiphonia & IV \\
\hline Delesseria & $\mathrm{TE}$ & Heterosiphonia & IV \\
\hline Delesseria & IV, VIII & Hildenbrandia & $\mathrm{X}$ \\
\hline Dermatolithon & VI & Hildenbrandia & $\mathrm{X}$ \\
\hline Digenea & IV & Hutchinsia & IV, N \\
\hline Dilsea & $\mathrm{TE}$ & Hypnea & VIII \\
\hline Dipterosiphonia & IV & Hypoglossum & IV \\
\hline Drachiella & IV & Iridaea & TE \\
\hline Dumontia & $\mathrm{TE}$ & Jania & VI \\
\hline Dumontia & TE & Jania & VI \\
\hline Epilithon & VI & Kallymenia & VIII \\
\hline Erythrochloropeltis & $\mathrm{V}$ & Laurencia & IV \\
\hline Erythrocladia & V & Laurencia & IV \\
\hline Erythropeltis & V & Lejolisia & IV \\
\hline Erythrotrichia & V & Liagora & $\mathrm{XI}$ \\
\hline Erytrocystis & IV & Litholepis & VI \\
\hline Falkenbergia & $\mathrm{N}$ & Lithophyllum & VI, N \\
\hline Fauchea & XIII & Lithophyllum & VI \\
\hline Feldmannophycus & $\mathrm{N}$ & Lithothamnion & VI \\
\hline Fosliella & VI & Lithothamnion & VI \\
\hline Fosliella & VI & Lomentaria & XIII \\
\hline Fucus & IV, VIII, IX, XIII, TE & Lophocladia & IV \\
\hline Furcelleria & VIII, N & Lophosiphonia & IV \\
\hline Furcellaria & VIII & Mastocarpus & VIII \\
\hline Gastroclonium & XIII & Melobesia & VI \\
\hline Gelidiella & VII & Melobesia & VI \\
\hline Gelidiocolax & VIII & Mesophyllum & VI \\
\hline Gelidium & VII, N & Mesothamnion & IV \\
\hline Gelidium & VII & Microcladia & IV \\
\hline Gigartina & VIII & Monosporus & IV \\
\hline Gigartina & XIII & Myriogramme & IV \\
\hline Goniolithon & VI & Myriogramme & IV \\
\hline Goniotrichum & XII & Nemalion & XI \\
\hline Gracilaria & IX & Neogoniolithon & VI, N \\
\hline Gracilaria & IX & Nitophyllum & IV \\
\hline Grateloupia & VIII, N & Ophidocladus & IV \\
\hline Griffithsia & IV, N & Osmundaria & IV \\
\hline
\end{tabular}




\begin{tabular}{|c|c|c|}
\hline Peyssonnelia & VIII & Spyridia \\
\hline Phycodrys & IV & Stenogramme \\
\hline Phyllophora & VIII & Stylonema \\
\hline Phyllophora & VIII, TE & Taenioma \\
\hline Phymatolithon & VI & Tenarea \\
\hline Platoma & VIII, N & Thuretella \\
\hline Platysiphonia & IV & Tiffaniella \\
\hline Platythamnion & IV & Titanoderma \\
\hline Plenosporium & IV & Trailliella \\
\hline Plocamium & VIII & Vickersia \\
\hline Plocamium & VIII & Vidalia \\
\hline Plumaria & TE & Wrangelia \\
\hline Pneophyllum & VI & Wurdemannia \\
\hline Pneophyllum & VI & \\
\hline Polyides & VIII, N & \\
\hline Polyneura & IV & ACKNOWLEDGEMENTS. This work was \\
\hline Polysiphonia & IV, $\mathrm{N}$ & financially supported by the Ministerio de Educación \\
\hline Polysiphonia & IV & y Ciencia (Spain), DGICYT project PB92-0415. \\
\hline Polystrata & VIII & Additional support was provided by a Universidad \\
\hline Porphyra & II & de Málaga pre-doctoral grant to M. Altamirano. Dr. \\
\hline Porphyropsis & II & Eric C. Henry (Department of Botany and Plant \\
\hline Pseudocrouania & IV & $\begin{array}{l}\text { Pathology, Oregon State University, Corvallis, } \\
\text { USA) kindly revised English style and grammar of }\end{array}$ \\
\hline Pterocladia & VII & the manuscript. \\
\hline Pterosiphonia & IV & \\
\hline Pterosiphonia & IV & \\
\hline Pterothamnion & IV & REFERENCES \\
\hline Ptilota & $\mathrm{TE}$ & REFEKLIVES \\
\hline Ptilothamnium & IV & \\
\hline Radicilingua & IV & AGARDH, C. A. -1823-Species algarum rite \\
\hline Rhodomela & TE & cognitae, cum synonimis, differentiis specificis \\
\hline Rhodomela & TE & et descriptionibus succintis. (Fucoideae, \\
\hline Rhodophyllis & VIII & Florideae, Ulvoideae). Sumtibus Ernestii \\
\hline Rhodymenia & XIII & Mauritii. Gryphiswald. 531pp. \\
\hline Rhodymenia & XIII & AGARDH, C. A. -1824- Systema algarum. Literis \\
\hline Rissoella & VIII & berlingianis. Lund. $312 \mathrm{pp}$ \\
\hline Rytiphlaea & IV & AGARDH, C. A. -1828-Species algarum rite \\
\hline Rytiphloea & IV & cognitae, cum synonimis, differentiis specificis \\
\hline Schizymenia & VIII, N & et descriptionibus succintis. (Lemanieae, \\
\hline Schottera & VIII & Ectocarpeae, Ceramieae). Gryphiae. 189 pp. \\
\hline Scinaia & $\mathrm{XI}, \mathrm{N}$ & AMO Y MORA, M. -1870- Flora Criptogámica de \\
\hline Scinaia & XI & la Península Ibérica que contiene la descripción \\
\hline Seirospora & IV & de las plantas acotiledóneas que crecen en \\
\hline Shalingia & V & España y Portugal distribuidas según el método \\
\hline Solieria & VIII & de Familias. Granada. \\
\hline Spermothamnion & IV & ARDISSONE, F. -1883-Phycologia Mediterranea. \\
\hline Spermothamnion & IV & Antica tipografia ferri di maj e malnati. Varese. \\
\hline Sphaerococcus & VIII & $515 \mathrm{pp}$ \\
\hline Sphaerococcus & VIII, IX, XIII & ARDRÉ, F. -1970-Contribution à l'étude des algues \\
\hline Sphondylothamnion & IV & marines du Portugal. Portugaliae Acta Biologica \\
\hline Spongites & VI & (Série B), 10: 137-532. \\
\hline
\end{tabular}


ATHANASIADIS, A. -1987-A survey of the seaweeds of the Aegean Sea with taxonomic studies on species of the tribe Antithamnieae (Rhodophyta). Akademisk avhanling. University of Gothenburg. Faculty of Natural Sciences.

AUGIER, H. et C.-F. BOUDOURESQUE -1971Découverte des cystocarpes de Feldmannophycus rayssiae (J. Feld. et G. Feld.) nov. gen. (Rhodophycées, Gigartinales). Bull. Soc. phycol. Fr., 16: 25-30.

BELLON, L. -1942- Las algas de la "Flora Boetica" inédita, de Clemente. Boletín del Ints. Esp. de Oc. Notas y Resúmenes, 2 (110). 93 pp.

BØRGESEN, F. -1929- Marine algae from the Canary Islands especially from Tenerife and Gran Canaria. III Rhodophyceae. Part II. Cryptonemiales, Gigatinales and Rhodymeniales. Det Kgl. Videnskabernes Selskab. Biologiske Meddelsr., 7: 97 pp + 4 pl.

BORNET, M. E. -1892 - Les algues de P.K.A. Schousboe, recoltés au Maroc et dans la Méditerranée de 1815 à 1829. Mém. Soc. Natl. Sci. Nat. et Math. Cherbourg, 28: 165-376.

CABIOCH, J., J.-Y. FLOCH, A. LE TOQUIN, C.-F. BOUDOURESQUE, A. MEINESZ et $M$. VERLAQUE -1992- Guide des algues des mers d'Europe. Delachaux et Niestlé, S. A., Neuchâtel. 231 pp.

CLEMENTE, S. DE R. -1807-Ensayo sobre las variedades de la vid común que vegetan en Andalucía, con un índice etimológico y tres listas de plantas en que se caracterizan varias especies nuevas. Madrid.

COLMEIRO, M. -1867- Enumeración de las Criptógamas de España y Portugal. [Algas]. Revista Progr. Ci. Exact., 18: 180-218, 360384, 431-441.

COLMEIRO, M. -1889-Enumeración y revisión de las plantas de la Península Hispano-Lusitana e islas Baleares. Madrid. Tomo 5: 875-1064.

CONDE, F. -1984- Catálogo de las algas macrobentónicas marinas de Málaga. Acta Bot. Malacitana, 9: 47-78.

CONDE, F. -1991- Catálogo y comentarios sobre el "complejo Audouinella" (Acrochaetiaceae, Rhodophyceae) en el Mediterráneo. Cryptogamie, Algol., 12: 163-170.

CONDE, F. -1992- Sobre la colección de algas del Herbario de la Sociedad Malagueña de Ciencias (s. XIX). Acta Bot. Malacitana, 17: 29-55.
CONDE, F. y J. SEOANE -1982- Nuevas aportaciones a la algología del litoral andaluz. Instituto de estudios almerienses. Homenaje almeriense al botánico Rufino Sagredo. Almería. pp. 83-97.

CONDE, F. y J. SOTO -1986- Notas corológicas del macrofitobentos de Andalucía (España). Acta Bot. Malacitana, 11: 9-16.

CONDE, F. y J. SOTO -1987- Nuevas contribuciones al estudio de la vegetación bentónica del litoral granadino. Actas VI Simp. Nac. Bot. Cript.: 3542.

CONDE, F., M. ALTAMIRANO y A. FLORESMOYA -1996- Notas corológicas del macrofitobentos de Andalucía (España). IV. Acta Bot. Malacitana, 21.

CONDE, F., A. FLORES-MOYA y J. J. VERAGONZÁLEZ -1990- Notas corológicas del macrofitobentos de Andalucía (España). II. Acta Bot. Malacitana, 15: 350-352.

CREMADES, J. -1993- Contribución al conocimiento de la obra ficológica de Simón de Rojas Clemente (1777-1827): tipificación de los nuevos nombres de su Ensayo. Anales Jard. Bot. Madrid, 51: 3-32.

CREMADES, J. y J. L. PÉREZ-CIRERA -1990Nuevas combinaciones de algas bentónicas marinas, como resultado del estudio del herbario de Simón de Rojas Clemente y Rubio (17771827). Anales Jard. Bot. Madrid, 47: 489-492.

DIXON, P. S. \& L. M. IR VINE -1977-Seaweeds of the British Isles. Vol. 1. Rhodophyta. Part 1. Introduction, Nemaliales, Gigartinales. Britsih Museum (NH). London. $252 \mathrm{pp}$.

FELDMANN, J. -1939- Les algues marines de la côte des Alberes. IV. Rhodophycées. Rev. Algol., 11: 247-330.

FELDMANN, J. -1942- Les algues marines de la côte des Alberes. IV. Rhodophycées. Trav. Algol., 1: 29-113.

FELDMANN, J. -1981- Clé des Polysiphonia des côtes françaises. Cryptogamie, Algol, 71-77.

FERNÁNDEZ, J. A., F. X. NIELL y F. CONDE 1983- Sobre la entidad taxonómica de los Gymnogongrus Martius, 1928, foliosos en las costas de Málaga y Cádiz (S. E. de la Península Ibérica). Inv. Pesq., 47: 161-165.

FLORES-MOYA, A. y F. CONDE -1992- Fenología y corología de Asparagopsis armata (Bonnemaisoniceae, Rhodophyta) en el 
Mediterráneo. Acta Bot. Malacitana, 17: 245-249. FLORES-MOYA, A., F. CONDE, A. SÁNCHEZ y M. ALTAMIRANO -1994- Notas corológicas del macrofitobentos de Andalucía (España). III. Acta Bot. Malacitana, 19: 211-213.

FLORES-MOYA, A., J. J. VERA-GONZÁLEZ y F. CONDE -1989- Contribución a la corología de las macroalgas marinas bentónicas del litoral malagueño. I. Acta Bot. Malacitana, 14: 199201.

FLORES-MOYA, A., J. SOTO, A. SÁNCHEZ, M. ALTAMIRANO, G. REYES and F. CONDE 1995a- Check-list of Andalucía (S. Spain) seaweeds. I. Phaeophyceae. Acta Bot. Malacitana, 20: 5-18.

FLORES-MOYA, A., J. SOTO, A. SÁNCHEZ, M. ALTAMIRANO, G. REYES and F. CONDE 1995b-Check-list of Andalucía (S. Spain) seaweeds. II. Chlorophyceae. Acta Bot. Malacitana, 20: 19-26.

FREDERICQ, S. and M. H. HOMMERSAND - 1989 Proposal of the Gracilariales ord. nov. (Rhodophyta) based on an analysis of the reproductive development of Gracilaria verrucosa. J. Phycol., 25: 213-227.

GIACCONE, G. -1972- Struttura, ecologia e corologia dei popolamenti a laminare dello Stretto di Messina e del Mare di Alboran. Mem. Biol. Mar. Ocean., 2: 35-79.

GONZÁLEZ-FRAGOSO, R. -1886- Plantas marinas de la costa de Cádiz. Anales Soc. Esp. Hist. Nat., 15: 117- 130.

GONZÁLEZ-FRAGOSO, R. -1893- Notas algológicas. Actas Soc. Esp. Hist. Nat., 22: 162-163.

GONZÁlEZ-GUERRERO, P. -1955- Contacto sociológico del Bangieto con el Enteromorphetum en el atlántico andaluz. Anales de la Real Academia de Farmacia, 21: 321-338.

IRVINE, L. M. -1983-Seaweeds of the British Isles. Vol. 1. Rhodophyta. Part 2A. Cryptonemiales (sensu stricto), Palmariales, Rhodymeniales. Britsih Museum (NH). London. 115 pp.

IRVINE, L. M. \& Y. M. CHAMBERLAIN -1994Seaweeds of the British Isles. Vol. 1.
Rhodophyta. Part 2B. Corallinales, Hildenbrandiales. Britsih Museum: HMSO. London. 276 pp.

KRAFT, G. T. and P. A. ROBINS -1985- Is the order Cryptonemiales (Rhodophyta) defensible? Phycologia, 24: 67-77.

LEMOINE, P. -1915- Calcareous algae. Repr. Dan. Ocean. Exp. 1908-10, 2.30 pp. +1 pl.

NAVARRO, M. J. y T. GALLARDO -1995Aportación al conocimiento de la flora bentónica marina de las costas de Huelva. Studia Botanica, 13: $51-60$.

NIELL, F. X. -1978- Nota sobre la presencia de talos monosexuados de Zanardinia prototypus Nardo en cubetas del sistema intermareal de la ría de Vigo. Inv. Pesq., 42: 25-31.

MAGGS, C. A. \& M. H. HOMMERSAND -1993Seaweeds of the British Isles. Vol. 1. Rhodophyta. Part 3A. Ceramiales. Britsih Museum: HMSO. London. 444 pp.

PÉREZ-CIRERA, J. L., J. CREMADES e I. BÁRBARA -1989- Grateloupia lanceola (Cryptonemiales, Rhodophyta) en las costas de la Península Ibérica: estudio morofológico y anatómico. Lazaroa, 11: 123-134.

PETERSEN, H. E. -1918- Algae (excl. calcareus algae). Repr. Dan. Ocean. Exp. 1908-10, 2(3): $1-20$.

PROLONGO, P. -1852- Plantas de Málaga y su término. In Martínez y Montes, V. Topografía médica de la ciudad de Málaga: 73-127. Málaga.

RULL LLUCH J., M. A. RIBERA et A. GOMEZ GARRETA - 1991- Quelques Rhodophyta intéressantes de la Méditerranée. Nova Hedwigia, 52: 149-159.

SAUVAGEAU, C. -1899- Sur les algues qui croissent sur les araignées de mer dans le golfe de Gascogne. C. R. Acad. Sci., 128: 696-698.

SCHOTTER, G. -1968-Recherches sur les Phyllophoracées. Bull. inst. océnogra. Monaco, 67: 1-99.

SEOANE, J. -1965- Estudios sobre las algas bentónicas en la costa sur de la Península Ibérica (litoral de Cádiz). Inv. Pesq., 29: 3-216.

SILVA, P. C. -1992- Nomenclatural notes on 
Clemente's Ensayo. Anales Jard. Bot. Madrid, 49: 163-170.

SOTO, J. - 1991- Datos sobre la flora y corología algal en el Mediterráneo de Andalucía (España): litoral de Granada. Anales Biol., 17: 105-108. SOTO, J. y F. CONDE -1989- Catálogo florístico de las algas bentónicas marinas del litoral de Almería (Sureste de España). Bot. Complutensis, 15: 61-83.

SOTO, J. y F. CONDE -1994- Aproximación a la vegetación algal dominada por Laurencia papillosa (C. Agardh) Greville, en la zona mediolitoral del Sureste de España. Acta Bot. Malacitana, 19:29-44.

TONI, G. B. DE e A. FORTI -1914- Seconda contribuzione alle flora algologica della Libia italiana. R. Com. Talassografico italiano, 70: 3-32.

VARO, J., J. RAMÍREZ y J. RENTERÍA - 1979 Estudio de la vegetación bentónica del litoral granadino. Acta Bot. Malacitana, 5: 79-98.
WEBB, P. B. -1853-Otia hispanica seu delectus plantarum rariorum aut nondum rite notarum per hispanicus sponte nascentium. Parissi. 52 pp. $+45 \mathrm{pl}$.

WOELKERLING, W. J. -1990- An introduction. In Cole, K. M. \& R. G. Sheat (eds.). The Biology of the Red Algae, pp. 1-6. Cambridge University Press, Cambridge.

Accepted for publication on May 1996

Author's addresses. F. Conde, A. Flores-Moya, M. Altamirano and A. Sánchez: Departamento de Biología Vegetal. Facultad de Ciencias. Universidad de Málaga. Campus de Teatinos s/n. E-29071 Málaga, Spain. E-mail: floresa@ccuma.sci.uma.es. J. Soto: Departamento de Biología Vegetal. Facultad de Biología. Campus del Espinardo. Universidad de Murcia. E-30100 Murcia, Spain. 